\title{
Lista de espécies dos Scarabaeinae (Coleoptera, Scarabaeidae) do Estado de Mato Grosso do Sul, Brasil
}

\author{
Fernando Z. Vaz-de-Mello', Leticia L. O. Bavutti', Carlos A. H. Flechtmann², \\ Anderson Puker $^{3}$ \& César M. A. Correa ${ }^{4}$
}

\begin{abstract}
1. Setor de Entomologia, Coleção Zoológica, Departamento de Biologia e Zoologia, Universidade Federal de Mato Grosso, Cuiabá, MT, Brasil. (vazdemello@gmail.com)

2. Departamento de Fitossanidade, Faculdade de Engenharia de Ilha Solteira, Universidade Estadual Paulista "Júlio de Mesquita Filho", Ilha Solteira, SP, Brasil. 3. Programa de Pós-Graduação em Entomologia, Departamento de Entomologia, Universidade Federal de Viçosa, Viçosa, MG, Brasil.

4. Programa de Pós-Graduação em Entomologia, Departamento de Entomologia, Universidade Federal de Lavras, Lavras, MG, Brasil.
\end{abstract}

Recebido 12 dezembro 2016

Aceito 6 fevereiro 2017

DOI: $10.1590 / 1678-4766 e 2017120$

ABSTRACT. Checklist of Scarabaeinae (Coeloptera: Scarabaeidae) from Mato Grosso do Sul State, Brazil. In this study we present an updated checklist of species of dung beetles of the subfamily Scarabaeinae, occurrying in Mato Grosso do Sul state, Brazil. One hundred and eighteen species are reported and associated with ecosystems or antropized habitat within the state of Mato Grosso do Sul.

KEYWORDS. Dung beetles, Pantanal, Cerrado, Atlantic Forest, Biota/MS Program.

RESUMO. Apresenta-se nesse estudo uma lista atualizada das espécies de besouros rola-bostas reportadas para o estado de Mato Grosso do Sul, Brasil. Cento e dezoito espécies são reportadas, e associadas aos ecossistemas ou hábitats antropizados em que ocorrem no estado.

PALAVRAS-CHAVE. Rola-bostas, Pantanal, Cerrado, Mata Atlântica, Programa Biota/MS.

Conhecidos popularmente como besouros "rolabostas", os escarabeíneos (Coleoptera, Scarabaeidae, Scarabaeinae) são detritívoros e utilizam principalmente fezes, carcaças e frutos em decomposição para alimentação (Halffter \& Matthews, 1966). Devido a este comportamento, desempenham diversos processos ecológicos como ciclagem de nitrogênio (HalfFter \& MATthews, 1966), aeração do solo (WATERHOUSE, 1974), supressão de parasitas (RIDSDILl-SMith \& HAYLES, 1990) e dispersão secundária de sementes (ANDRESEN, 2002), entre outras funções (ver revisão em NichOLs et al., 2008). Têm sido sugeridos e usados no biomonitoramento de áreas de proteção ambiental e na avaliação dos efeitos de qualquer atividade humana realizada nessas áreas (ver revisão em NicHOLS et al., 2007).

A última listagem das espécies de Scarabaeidae citadas para o Brasil foi realizada por VAZ-DE-MeLlo (2000), que reportou 618 espécies e estimou que, com a realização de novos levantamentos em regiões que não foram devidamente amostradas, utilizando metodologias distintas e modernas, este número poderia ultrapassar 1.200 espécies. Neste mesmo estudo, foram mencionadas para o estado do Mato Grosso do Sul 75 espécies, sendo duas endêmicas. Dados atualizados até 2011 (F. Z. Vaz-de-Mello, dados inéditos) reportam agora mais de 700 espécies registradas para o país, que conta certamente com mais de mil espécies conhecidas em coleções, se adicionadas as ainda não identificadas.

Neste estudo foram revisados os registros de literatura e a identificação das espécies reportadas para o estado do Mato Grosso do Sul, atualizando a lista das espécies de Scarabaeinae para o estado e discutindo as lacunas existentes em termos de amostragem no estado, contribuindo para indicar áreas de estudos futuros.

\section{MATERIAL E MÉTODOS}

O estudo foi realizado através de pesquisa bibliográfica em virtualmente toda a literatura publicada sobre Scarabaeinae até meados do ano de 2012. Essa compilação incluiu trabalhos taxonômicos, faunísticos, ecológicos e de interesse econômico que citassem ocorrência de espécies identificadas de Scarabaeinae em Mato Grosso do Sul (Boucomont, 1928; Luederwaldt, 1929, 1931a,b; PessôA, 1934; Pessôa \& Lane, 1936, 1941; Pereira, 1942, 1946, 1949, 1953; Pereira \& D'ANDRETTA, 1955; HalfFter et al., 1960; FLECHTMANn et al., 1995a,b,c,d, 2009; GÉNIER, 1996, 2009, 2010; Rodrigues \& Flechtmann, 1997; Koller et al., 1999, 2007; AIDAR et al., 2000; LOPES, 2000; RODRIGUES et al., 2001a,b, 2010; CANHEDo, 2004, 2006; LouZADA et al., 2007; VAZ-DE-MELLO, 2007a,b, 2008; RodRIGUES, 2008; 
Mesquita Filho, 2009; VaZ-De-Mello \& Grossi 2010; Авот et al., 2012; NuNES, 2012), tendo sido tão exaustiva quanto possível. Todos os registros previamente existentes foram revisados quanto a suas identificações (quando possível inclusive com base em exemplares-testemunho), descartandose suspeitas fortes de identificações errôneas e corrigindo-se as anteriormente publicadas cuja identificação correta pôde ser confirmada pelo exame de exemplares.

Foram também listados os registros sulmatogrossenses das duas coleções que possuem os maiores acervos deste estado, o Setor de Entomologia da Coleção Zoológica da Universidade Federal de Mato Grosso (CEMT, curador F.Z. Vaz-de-Mello) e o Museu de Entomologia da Faculdade de Engenharia de Ilha Solteira, da Universidade Estadual Paulista "Júlio de Mesquita Filho" (MEFEIS, curador C.A.H. Flechtmann). Registros isolados conhecidos de outras coleções foram também incorporados.

As espécies citadas no presente estudo puderam ser também associadas aos ecossistemas naturais presentes no estado (Cerrado, Mata Atlântica, Pantanal ou Chaco) ou a um tipo de ecossistema antrópico (pastagem formada), com base em dados de coleções ou distribuição geográfica conhecida.

\section{RESULTADOS E DISCUSSÃO}

Até o ano de 2000 tinham sido registradas 75 espécies de besouros da subfamília Scarabaeinae para o estado do Mato Grosso do Sul. Foram contabilizadas até o momento 117 espécies de besouros rola-bosta identificadas para o estado (Tab. I). Como a sistemática supragenérica do grupo

Tab. I. Espécies de besouros Scarabaeinae registradas do Estado de Mato Grosso do Sul, Brasil e seus hábitats ou ecossistemas de ocorrência. Dados baseados em literatura publicada até 2011 e em espécimes depositados em CEMT e MEFEIS (ver metodologia para detalhes) (Pas, pastagem formada; C, Cerrado; MA, Mata Atlântica; Pan, Pantanal; Ch, Chaco).

\begin{tabular}{|c|c|c|c|c|c|}
\hline \multirow{2}{*}{ Espécie } & \multicolumn{5}{|c|}{ Ocorrência } \\
\hline & Pas & Cer & MA & Pan & $\mathrm{Ch}$ \\
\hline Agamopus unguicularis (Harold, 1883) & $\mathrm{x}$ & $\mathrm{x}$ & & & \\
\hline Agamopus viridis Boucomont, 1928 & $\mathrm{x}$ & $\mathrm{x}$ & & & \\
\hline Anomiopus birai Canhedo, 2006 & $\mathrm{x}$ & & & & \\
\hline Anomiopus mourai Canhedo, 2006 & & $\mathrm{x}$ & & & \\
\hline Anomiopus myrmidon (Westwood, 1842) & & & $\mathrm{x}$ & & \\
\hline Anomiopus pereirai (Martínez, 1955) & $\mathrm{x}$ & $\mathrm{x}$ & & & \\
\hline Anomiopus tuberifrons Canhedo, 2004 & & $\mathrm{x}$ & & & \\
\hline Anomiopus virescens Westwood, 1842 & $\mathrm{x}$ & $\mathrm{x}$ & & & \\
\hline Ateuchus apicatus (Harold,1867) & $\mathrm{x}$ & $\mathrm{x}$ & & & \\
\hline Ateuchus latus (Boucomont, 1928) & $\mathrm{x}$ & & & $\mathrm{x}$ & \\
\hline Ateuchus pauperatus (Germar, 1824) & $\mathrm{x}$ & $\mathrm{x}$ & & & \\
\hline Ateuchus puncticollis (Harold, 1867) & & $\mathrm{x}$ & & & \\
\hline Ateuchus pygidialis (Harold, 1868) & $\mathrm{x}$ & $\mathrm{x}$ & & & \\
\hline Ateuchus striatulus (Preudhomme de Borre, 1886) & $\mathrm{x}$ & $\mathrm{x}$ & & & \\
\hline Ateuchus viridimicans (Boucomont, 1935) & $\mathrm{x}$ & $\mathrm{x}$ & & & \\
\hline Ateuchus vividus (Germar, 1823) & $\mathrm{x}$ & $\mathrm{x}$ & & & \\
\hline Bolbites onitoides Harold, 1868 & & & & & $\mathrm{x}$ \\
\hline Canthidium angulicolle Balthasar, 1939 & & & & $\mathrm{x}$ & \\
\hline Canthidium barbacenicum Preudhomme de Borre, 1886 & $\mathrm{x}$ & $\mathrm{x}$ & & $\mathrm{x}$ & $\mathrm{x}$ \\
\hline Canthidium breve (Germar, 1824) & & & & $\mathrm{x}$ & \\
\hline Canthidium decoratum (Perty, 1830) & $\mathrm{x}$ & & & & \\
\hline Canthidium dispar Harold, 1867 & & & $\mathrm{x}$ & & \\
\hline Canthidium viride Lucas, 1859 & $\mathrm{x}$ & $\mathrm{x}$ & & $\mathrm{x}$ & \\
\hline Canthon apicalis Lucas, 1859 & & & & $\mathrm{x}$ & $\mathrm{x}$ \\
\hline Canthon chalybaeus Blanchard, 1846 & $\mathrm{x}$ & $\mathrm{x}$ & $\mathrm{x}$ & & \\
\hline Canthon curvodilatatus Schmidt, 1920 & & & & $\mathrm{x}$ & $\mathrm{x}$ \\
\hline Canthon denticulatus Schmidt, 1922 & $\mathrm{x}$ & & & & \\
\hline Canthon deplanatus Harold, 1868 & $\mathrm{x}$ & & & & \\
\hline Canthon dives Harold, 1868 & $\mathrm{x}$ & & & & \\
\hline Canthon edentulus Harold, 1868 & $\mathrm{x}$ & & & $\mathrm{x}$ & $\mathrm{x}$ \\
\hline Canthon histrio (Lepelletier de Saint-Fargeau \& Audinet-Serville, 1828) ${ }^{1}$ & $\mathrm{x}$ & $\mathrm{x}$ & $\mathrm{x}$ & $\mathrm{x}$ & $\mathrm{x}$ \\
\hline Canthon laminatus Balthasar, 1939 & $\mathrm{x}$ & & $\mathrm{x}$ & & \\
\hline Canthon lituratus (Germar, 1824) & $\mathrm{x}$ & & $\mathrm{x}$ & & \\
\hline Canthon maldonadoi Martínez, 1951 & & $\mathrm{x}$ & $\mathrm{x}$ & $\mathrm{x}$ & $\mathrm{x}$ \\
\hline Canthon mutabilis Lucas, 1859 & $\mathrm{x}$ & $\mathrm{x}$ & & $\mathrm{x}$ & \\
\hline Canthon oliverioi (Pereira \& Martínez, 1956) & & & $\mathrm{x}$ & & \\
\hline Canthon ornatus ornatus Redtenbacher, 1867 & $\mathrm{x}$ & $\mathrm{x}$ & & $\mathrm{x}$ & $\mathrm{x}$ \\
\hline Canthon planus Lucas, 1859 & $\mathrm{x}$ & & & & \\
\hline Canthon quadratus Blanchard, 1846 & & & & $\mathrm{x}$ & \\
\hline Canthon quinquemaculatus Castelnau, 1840 & & $\mathrm{x}$ & $\mathrm{x}$ & $\mathrm{x}$ & $\mathrm{x}$ \\
\hline Canthon smaragdulus (Fabricius, 1781) & & & $\mathrm{x}$ & & \\
\hline Canthon substriatus Harold, 1868 & $\mathrm{x}$ & & & & \\
\hline
\end{tabular}


Tab. I. Cont.

\begin{tabular}{|c|c|c|c|c|c|}
\hline \multirow{2}{*}{ Espécie } & \multicolumn{5}{|c|}{ Ocorrência } \\
\hline & Pas & Cer & MA & Pan & $\mathrm{Ch}$ \\
\hline Canthon unicolor Blanchard, 1843 & & $\mathrm{x}$ & & $\mathrm{x}$ & $\mathrm{x}$ \\
\hline Canthon virens (Mannerheim, 1829) & $\mathrm{x}$ & $\mathrm{x}$ & & & \\
\hline Coprophanaeus cyanescens d'Olsoufieff, 1924 & $\mathrm{X}$ & $\mathrm{X}$ & $\mathrm{X}$ & $\mathrm{x}$ & $\mathrm{x}$ \\
\hline Coprophanaeus ensifer (Germar, 1824) & $\mathrm{x}$ & $\mathrm{x}$ & & & \\
\hline Coprophanaeus horus (Waterhouse, 1891) & & $\mathrm{x}$ & & & \\
\hline Coprophanaeus milon (Blanchard, 1846) & & & & $\mathrm{x}$ & \\
\hline Coprophanaeus pessoai (Pereira, 1949) & & $\mathrm{x}$ & & & \\
\hline Coprophanaeus spitzi (Pessôa, 1935) & $\mathrm{x}$ & $\mathrm{x}$ & & & \\
\hline Deltochilum elongatum Felsche, 1907 & & & & $\mathrm{x}$ & $\mathrm{x}$ \\
\hline Deltochilum enceladum Kolbe, 1893 & $\mathrm{x}$ & $\mathrm{x}$ & & & \\
\hline Deltochilum icaroides Balthasar, 1939 & & & $\mathrm{x}$ & $\mathrm{x}$ & \\
\hline Deltochilum pseudoicarus Balthasar, 1939 & $\mathrm{x}$ & $\mathrm{x}$ & & $\mathrm{x}$ & \\
\hline Deltorhinum bilobatum Génier, 2010 & & $\mathrm{x}$ & & & \\
\hline Dendropaemon denticollis Felsche, 1909 & $\mathrm{x}$ & & & & \\
\hline Dendropaemon viridipennis (Laporte, 1830) & & $\mathrm{x}$ & & & \\
\hline Diabroctis mimas (Linnaeus, 1758) & $\mathrm{x}$ & $\mathrm{x}$ & & & \\
\hline Diabroctis mirabilis (Harold, 1877) & $\mathrm{x}$ & $\mathrm{x}$ & & & \\
\hline Dichotomius bicuspis (Germar, 1824) & $\mathrm{x}$ & $\mathrm{x}$ & & & \\
\hline Dichotomius bos (Blanchard, 1846) ${ }^{2}$ & $\mathrm{x}$ & $\mathrm{x}$ & & $\mathrm{x}$ & \\
\hline Dichotomius carbonarius (Mannerheim, 1929) & $\mathrm{x}$ & $\mathrm{x}$ & $\mathrm{x}$ & $\mathrm{x}$ & $\mathrm{x}$ \\
\hline Dichotomius crinicollis (Germar, 1824) & $\mathrm{x}$ & & & & \\
\hline Dichotomius cuprinus (Felsche, 1901) & $\mathrm{x}$ & & & & \\
\hline Dichotomius depressicollis (Harold, 1867) & & & $\mathrm{x}$ & & \\
\hline Dichotomius eucranioides Pereira \& D’Andretta, 1955 & $\mathrm{x}$ & $\mathrm{x}$ & & & \\
\hline Dichotomius glaucus (Harold, 1869) & $\mathrm{x}$ & $\mathrm{x}$ & & & \\
\hline Dichotomius luctuosioides (Harold, 1869) & $\mathrm{x}$ & & & $\mathrm{x}$ & \\
\hline Dichotomius nisus (Olivier, 1789) & $\mathrm{x}$ & $\mathrm{x}$ & & $\mathrm{x}$ & $\mathrm{x}$ \\
\hline Dichotomius opacipennis (Luederwaldt, 1931) & $\mathrm{x}$ & $\mathrm{x}$ & & $\mathrm{x}$ & $\mathrm{x}$ \\
\hline Dichotomius semiaeneus (Germar, 1824) & $\mathrm{x}$ & $\mathrm{x}$ & & & \\
\hline Dichotomius triangulariceps (Blanchard, 1846) & $\mathrm{x}$ & $\mathrm{x}$ & & & \\
\hline Dichotomius zikani (Luederwaldt, 1922) & $\mathrm{x}$ & $\mathrm{x}$ & & & \\
\hline Digitonthophagus gazella (Fabricius, 1787$)^{3}$ & $\mathrm{x}$ & $\mathrm{x}$ & & & \\
\hline Eurysternus aeneus Génier, 2009 & & & & $\mathrm{x}$ & $\mathrm{x}$ \\
\hline Eurysternus caribaeus (Herbst, 1789) & $\mathrm{x}$ & $\mathrm{x}$ & $\mathrm{x}$ & $\mathrm{x}$ & $\mathrm{x}$ \\
\hline Eurysternus jessopi Martínez, 1988 & & $\mathrm{x}$ & & & $\mathrm{x}$ \\
\hline Eurysternus navajasi Martínez, 1988 & & $\mathrm{x}$ & & & \\
\hline Eurysternus nigrovirens Génier, 2009 & $\mathrm{x}$ & $\mathrm{x}$ & $\mathrm{x}$ & $\mathrm{x}$ & \\
\hline Eurysternus parallelus Castelnau, 1840 & $\mathrm{x}$ & & $\mathrm{x}$ & & \\
\hline Eurysternus plebejus Harold, 1880 & & & & $\mathrm{x}$ & \\
\hline Eutrichillum hirsutum (Boucomont, 1928) & $\mathrm{x}$ & $\mathrm{x}$ & $\mathrm{x}$ & & \\
\hline Genieridium bidens (Balthasar, 1938) & $\mathrm{x}$ & $\mathrm{x}$ & & & \\
\hline Genieridium cryptops (Arrow, 1913) & & $\mathrm{x}$ & & & \\
\hline Gromphas inermis Harold, 1869 & $\mathrm{x}$ & & & $\mathrm{x}$ & \\
\hline Isocopris foveolatus (Luederwaldt, 1931) & & $\mathrm{x}$ & & & $\mathrm{x}$ \\
\hline Isocopris inhiatus (Germar, 1824) & $\mathrm{x}$ & $\mathrm{x}$ & & & \\
\hline Isocopris tarsalis (Luederwaldt, 1931) & & $\mathrm{x}$ & & & $\mathrm{x}$ \\
\hline Malagoniella puncticollis (Blanchard, 1846) & & & & $\mathrm{x}$ & $\mathrm{x}$ \\
\hline Malagoniella astyanax (Olivier, 1789) & $\mathrm{x}$ & & $\mathrm{x}$ & & \\
\hline Malagoniella punctatostriata (Blanchard, 1846) & & $\mathrm{x}$ & & $\mathrm{x}$ & $\mathrm{x}$ \\
\hline Malagoniella aeneicollis (Waterhouse, 1890) & $\mathrm{x}$ & $\mathrm{x}$ & $\mathrm{x}$ & & \\
\hline Ontherus appendiculatus (Mannerheim, 1829) & $\mathrm{x}$ & $\mathrm{x}$ & & $\mathrm{x}$ & \\
\hline Ontherus azteca Harold, 1869 & & $\mathrm{x}$ & $\mathrm{x}$ & & $\mathrm{x}$ \\
\hline Ontherus carinicollis Luederwaldt, 1931 & $\mathrm{x}$ & $\mathrm{x}$ & & & \\
\hline Ontherus dentatus Luederwaldt, 1930 & $\mathrm{x}$ & $\mathrm{x}$ & & $\mathrm{x}$ & \\
\hline Ontherus digitatus Harold, 1868 & $\mathrm{x}$ & $\mathrm{x}$ & & $\mathrm{x}$ & \\
\hline Ontherus erosioides Luederwaldt, 1930 & & $\mathrm{x}$ & & $\mathrm{x}$ & \\
\hline Ontherus sulcator (Fabricius, 1775) & $\mathrm{x}$ & & $\mathrm{x}$ & $\mathrm{x}$ & \\
\hline Ontherus ulcopygus Génier, 1996 & & $\mathrm{x}$ & & & \\
\hline Ontherus zikani Luederwaldt, 1930 & $\mathrm{x}$ & & $\mathrm{x}$ & & \\
\hline
\end{tabular}


Tab. I. Cont.

\begin{tabular}{|c|c|c|c|c|c|}
\hline \multirow{2}{*}{ Espécie } & \multicolumn{5}{|c|}{ Ocorrência } \\
\hline & Pas & Cer & MA & Pan & $\mathrm{Ch}$ \\
\hline Oxysternon striatopunctatum d'Olsoufieff, 1924 & & $\mathrm{x}$ & & & \\
\hline Phanaeus kirbyi Vigors, 1825 & $\mathrm{x}$ & $\mathrm{x}$ & & & \\
\hline Phanaeus palaeno Blanchard, 1846 & $\mathrm{x}$ & $\mathrm{x}$ & & $\mathrm{x}$ & \\
\hline Pseudocanthon xanthurus (Blanchard, 1845) & $\mathrm{x}$ & $\mathrm{x}$ & & $\mathrm{x}$ & $\mathrm{x}$ \\
\hline Sulcophanaeus menelas (Castelnau, 1840) & $\mathrm{x}$ & & & $\mathrm{x}$ & \\
\hline Trichillidium quadridens Arrow, 1932 & & & & $\mathrm{x}$ & $\mathrm{x}$ \\
\hline Trichillum adjunctum Martínez, 1967 & & $\mathrm{x}$ & & & \\
\hline Trichillum externepunctatum Preudhomme de Borre, 1886 & $\mathrm{x}$ & $\mathrm{x}$ & $\mathrm{x}$ & $\mathrm{x}$ & $\mathrm{x}$ \\
\hline Uroxys epipleuralis (Boucomont, 1928) & $\mathrm{x}$ & $\mathrm{x}$ & & & \\
\hline Zonocopris gibbicollis (Harold, 1868) & & & & $\mathrm{x}$ & \\
\hline
\end{tabular}

1. Frequentemente citado como Canthon septemmaculatus. 2. Em trabalhos mais antigos, citado como seu sinônimo Dichotomius anaglypticus.

3. Em trabalhos mais antigos, citado no gênero Onthophagus

é atualmente confusa e está em rápida mudança (VAZ-DEMELlo, 2007a), desconsideramos aqui as tribos. Esse é um número de espécies pequeno para o tamanho e condições climáticas do estado, bem menor que o número de espécies reportadas para o estado de São Paulo, por exemplo (208 espécies; F. Z. Vaz-de-Mello, dados inéditos).

A grande maioria das espécies registradas do estado está em áreas de Cerrado ou pastagens artificiais, havendo importantes componentes ocorrendo na Mata Atlântica (com grande divergência em relação às demais formações) e no Pantanal. Porém, a desuniformidade dos registros de localidades disponíveis em relação à área total do estado impede análises mais precisas.

A única espécie não nativa ocorrente no estado é Digitonthophagus gazella, que é de origem africana, introduzida no Brasil no final dos anos 1980 com a intenção de contribuir ao controle biológico de parasitos de gado e auxiliar na incorporação de massas fecais (MiRANDA et al., 1990).

Anomiopus birai e Bolbites onitoides não têm registros de outros estados brasileiros, mas ocorrem em países limítrofes (respectivamente Paraguai, Argentina, Bolívia e Uruguai) (CANHEDO, 2006; VAZ-DE-MELlo \& GROSSI, 2010). Duas outras espécies não tem registros de outros estados ou países: Ateuchus latus e Dichotomius eucranioides. A primeira foi descrita originalmente de Corumbá e muito provavelmente ocorre também no extremo sul de MT e na Bolívia, mas por pertencer a um gênero pouco estudado, o registro não foi ainda efetuado.

A segunda, Dichotomius eucranioides, necessita atenção especial quanto à conservação, por ser uma espécie braquíptera com área de distribuição geográfica restrita à margem oeste do Rio Paraná (NUNES, 2012). Foi registrada apenas ao longo de uma faixa de $70 \mathrm{~km}$ de mata ciliar e áreas de cerrado limítrofes do rio Paraná, região que teve sua vegetação removida por muitas décadas e tem pouquíssimos remanescentes de vegetação nativa (R. V. Nunes e F. Z. Vazde-Mello, dados inéditos).

No estado de Mato Grosso do Sul pesquisadores de três instituições têm publicado ou têm em andamento trabalhos com Scarabaeinae: Empresa Brasileira de Pesquisa
Agropecuária (EMBRAPA-CNPGC em Campo Grande), Universidade Estadual de Mato Grosso do Sul (UEMS em Aquidauana), e Universidade Federal da Grande Dourados (UFGD em Dourados), em geral com aspectos econômicos ou ecológicos de espécies associadas a pastagens, ou com faunística e biogeografia, e sempre em colaboração com taxônomos de outras instituições. Há pequenas coleções resultantes de trabalhos feitos no estado na Universidade Federal de Mato Grosso do Sul (UFMS-Campo Grande) e na UFGD-Dourados. Além disso, pesquisadores de outros estados têm trabalhado com fauna de Scarabaeinae de Mato Grosso do Sul, muito especialmente da Faculdade de Engenharia de Ilha Solteira, Universidade Estadual Paulista "Júlio de Mesquita Filho" (FEIS-UNESP, Ilha Solteira), da Universidade Federal de Lavras (UFLA-Lavras) e da Universidade Federal de Mato Grosso (UFMT- Cuiabá). Isso pode se ver refletido na literatura citada.

O estado está relativamente bem amostrado em algumas regiões do Pantanal, em seu extremo leste, junto ao Rio Paraná (próximo a Selvíria) e, no caso de pastagens, em Campo Grande, Selvíria e Aquidauana. Coletas recentes em várias localidades da Serra da Bodoquena e seu entorno trouxeram dezenas de espécies previamente não citadas para o estado, sendo as demais regiões virtualmente vazias de coletas. Projetos em andamento associando pesquisadores de diversas instituições devem trazer novos dados sobre as áreas ainda inexploradas do estado.

Agradecimentos. À Fundação de Apoio ao Desenvolvimento do Ensino, Ciências e Tecnologia do Estado de Mato Grosso do Sul (Fundect) e a Superintendência de Ciências e Tecnologia do Estado de Mato Grosso do Sul (Sucitec/MS) pelo convite de participação neste fascículo especial da Iheringia, Série Zoologia e o suporte financeiro para sua publicação. FZVM agradece ao CNPq (Proc. 304925/2010-1, 302997/2013-0, 405697/2013-9, 484035/2013-4), e a Maria Eugênia Carvalho do Amaral, Frederico Santos Lopes, Júlio Louzada, Masao Uetanabaro, Fábio Roque, Marcelo B. Pessoa, Vinícius Lopes, Josué Raizer, Wilson W. Koller, Marino Rodrigues, Manoel A. Uchôa Fernandes, Carlos Flechtmann e Lúcio A. O. Campos por anos de apoio nas formas de logística, espécimes ou trabalho duro para coletas em MS. CAHF agradece aos funcionários do Setor de Bovinocultura da Fazenda da UNESP, pelos anos de suporte à condução dos experimentos lá desenvolvidos. AP agradece ao CNPq (processo 140989/2011-0) pela bolsa de estudos concedida e ao Programa de Pós-Graduação em Entomologia da Universidade Federal de Viçosa. CMAC agradece ao CNPq 
(processo140741/2015-1) pela bolsa de estudos concedida e ao Programa de Pós-Graduação em Entomologia da Universidade Federal de Lavras.

\section{REFERÊNCIAS BIBLIOGRÁFICAS}

Abot, A. R.; Puker, A.; Taira, T. L.; Rodrigues, S. R.; Korasaki, V. \& Oliveira, H. N. 2012. Abundance and diversity of coprophagous beetles (Coleoptera: Scarabaeidae) caught with a light trap in a pasture area of the Brazilian Cerrado. Studies on Neotropical Fauna and Environment 47:53-60.

Aidar, T.; Koller, W. W.; Rodrigues, S. R.; Correa, A. M.; Silva, J. C. C.; Balta, O. S.; Oliveira, J. M. \& Oliveira, V. L. 2000. Besouros coprófagos (Coleoptera: Scarabaeidae) coletados em Aquidauana, MS, Brasil. Anais da Sociedade Entomológica do Brasil 29(4):817-820.

ANDRESEN, E. 2002. Dung beetles in a Central Amazonian rainforest and their ecological role as secondary seed dispersers. Ecological Entomology 27:257-270.

Boucomont, A. 1928. Coprophages d'Amerique du Sud nouveaux ou peu connus. Bulletin de la Société Entomologique de France 1928:186194.

Canhedo, V. L. 2004. Anomiopus Westwood (Coleoptera: Scarabaeidae): novas espécies do grupo virescens. Revista Brasileira de Entomologia 48(4):449-458

CAnHedo, V. L. 2006. Revisão taxonômica do Gênero Anomiopus Westwood, 1842 (Coleoptera, Scarabaeidae, Scarabaeidae). Arquivos de Zoologia 37(4):349-502.

Flechtmann, C. A. H.; Rodrigues, S. R. \& Seno, M. C. Z. 1995a. Controle biológico da mosca-dos-chifres (Haematobia irritans irritans) em Selvíria, Mato Grosso do Sul. 1. Metodologia de estudo e seleção de fauna fimícola de insetos. Revista Brasileira de Entomologia 39(1):1-11.

Flechtmann, C. A. H.; Rodrigues, S. R. \& Seno, M. C. Z. 1995b. Controle biológico da mosca-dos-chifres (Haematobia irritans irritans) em Selvíria, Mato Grosso do Sul. 3. Levantamento de espécies fimícolas associadas à mosca. Revista Brasileira de Entomologia 39(2):249-258.

Flechtmann, C. A. H.; Rodrigues, S. R. \& Seno, M. C. Z. 1995c. Controle biológico da mosca-dos-chifres (Haematobia irritans irritans) em Selvíria, Mato Grosso do Sul. Comparação entre métodos de coleta de besouros coprófagos (Scarabaeidae). Revista Brasileira de Entomologia 39(2):259-276.

Flechtmann, C. A. H.; Rodrigues, S. R. \& Seno, M. C. Z. 1995d. Controle biológico da mosca-dos-chifres (Haematobia irritans irritans) em Selvíria, Mato Grosso do Sul. Seleção de besouros coprófagos. Revista Brasileira de Entomologia 39(2):277-286.

Flechtmann, C. A. H.; TABet, V. G. \& Quintero, I. 2009. Influence of carrion smell and rebaiting time on the efficiency of pitfall traps to dung beetle sampling. Entomologia Experimentalis et Applicata 132(3):211-217.

GÉNIER, F. 1996. A revision the Neotropical genus Ontherus. Memoirs of the Entomological Society of Canada 170:1-169.

GÉnIER, F. 2009. Le genre Eurysternus Dalman, 1824 révision taxonomique et clés de détermination illustrées. Bulgaria, Pensoft Publishers, Sofia. 430 p.

GÉnIER, F. 2010. A Review of the Neotropical dung beetle genera Deltorhinum Harold, 1869, and Lobidion gen. nov. (Coleoptera: Scarabaeidae: Scarabaeinae). Zootaxa 2693:35-48.

Halffter, G. \& Matthews, E. G. 1966. The natural history of dung beetles of the Subfamily Scarabaeinae (Coleoptera, Scarabaeidae). Folia Entomologica Mexicana 12-14:1-312.

Halffter, G.; Pereira, F. S. \& Martínez, A. 1960. Megathopa astyanax (Olivier) y formas afines (Coleóptera Scarabaeidae) Ciencia (México) 20:202-204.

Koller, W. W.; Gomes, A.; Rodrigues, S. R. \& Alves, R. G. O. 1999. Besouros Coprófagos (Coleoptera: Scarabaeidae) Coletados em Campo Grande, MS, Brasil. Anais da Sociedade Entomológica do Brasil 28(3):403-412

Koller, W.; Gomes, A.; Rodrigues, S. R. \& Goiozo, P. F. I. 2007 Scarabaeidae e Aphodiidae coprófagos em pastagens cultivadas em áreas do cerrado sul-mato-grossense. Revista Brasileira de Zoociências 9(1):81-93

Lopes, V. A. 2000. Seleção de habitat e estrutura de comunidades de coleópteros copronecrófagos da família Scarabaeidae. Dissertação de Mestrado. Campo Grande, Universidade Federal de Mato Grosso do Sul.
Louzada, J. N. C.; Lopes, F. S. \& VaZ-De-Mello, F. Z. 2007. Structure and composition of a dung beetle community (Coleoptera, Scarabaeinae) in a small forest patch from Brazilian Pantanal. Revista Brasileira de Zoociências 9(2):199-203.

Luederwaldt, H. 1929. As especies brasileiras do genero Pinotus. Revista do Museu Paulista 16:603-775.

LuederwaldT, H. 1931a. Tres especies novas de Pinotus, supplementos a Memoria "As especies Brasileiras do genero Pinotus" na "Revista do Museu Paulista” Tomo XVI, 1929, P. 603 SS. Revista do Museu Paulista 17:353-361.

Luederwaldt, H. 1931b. O genero Ontherus (Coleopt.) (Lamellic-Coprid.Pinot.) com uma chave, para a determinação dos Pinotides americanos. Revista do Museu Paulista 17:363-422.

Mesquita FiLho, W. 2009. Determinação de horário de vôo e fatores que o influenciam, em Scarabaeidae coprófagos diurnos e noturnos em Selvíria, MS. Dissertação de Mestrado. Ilha Solteira, Universidade Estadual Paulista.

Miranda, C. H. B.; NAScimento, Y.A. \& Bianchin, I. 1990. Desenvolvimento de um programa integrado de controle de nematódeos e a mosca-doschifres na região dos cerrados. Fase 3. Potencial de Onthophagus gazella no enterrio de fezes bovinas. EMBRAPA-CNPGC. Pesquisa em Andamento 40:1-5.

Nichols, E.; Larsen, T.; Spector, S.; Davis, A. L.; Escobar, F.; Favila, M. \& VulineC, K. 2007. Global dung beetle response to tropical forest modification and fragmentation: A quantitative literature review and meta-analysis. Biological Conservation 137:1-19.

Nichols, E.; Spector, S.; Louzada, J.; Larsen, T.; Amezquita, S. \& FAVILA, M. E. 2008. The Scarabaeinae Research Network. Ecological Functions and ecosystems services provided by Scarabaeinae dung beetles. Biological Conservation 141:1461-1474.

NunES, R. V. 2012. Review of the brachypterous species of the genus Dichotomius Hope, 1838 (Coleoptera: Scarabaeidae; Scarabaeinae). Dissertação de Mestrado. Cuiabá, Universidade Federal de Mato Grosso.

Pereira, F. S. 1942. Pinotus da seção semiaeneus. Arquivos do Museu Paranaense 2:35-60.

Pereira, F. S. 1946. Escarabeideos americanos II. In: Livro de Homenagem a Romualdo Ferreira d'Almeida. São Paulo, Sociedade Brasileira de Entomologia. p. 289-294

PereIRA, F. S. 1949. O subgenero Metallophanaeus (ColeópteraScarabaeidae). Arquivos do Museu Paranaense 7:217-230.

Pereira, F. S. 1953 Notas sinonímicas (Coleoptera Scarabaeidae). Dusenia 4(5,6):387-402.

Pereira, F. S. \& D’Andretta, M. A. V. 1955. Novos escarabeídeos e novas sinonímias (Coleoptera Scarabaeidae). Papéis Avulsos do Departamento de Zoologia 12(11):247-264.

PessôA, S. B. 1934. Contribuição para o conhecimento das especies brasileiras do genero Phanaeus (Col. Scarabaeidae). Annaes da Faculdade de Medicina de São Paulo 1934:279-314.

PessôA, S. B. \& Lane, J. 1936. Sobre os coleópteros do genero Dendropaemon Perty, de São Paulo e regiões vizinhas. Revista de Biologia e Hygiene 7(2):89-93.

PessôA, S. B. \& Lane, J. 1941 Coleópteros necróphagos de interesse médico-legal. Ensaio monografico sobre a família Scarabaeidae de São Paulo e regioes vizinhas. Arquivos de Zoologia do Estado de São Paulo 2:389-504.

Ridsdill-Smith, T. J. \& Hayles, L. 1990. Stages of bush fly, Musca vetustissima (Diptera: Muscidae), killed by scarabaeine dung beetles (Coleoptera: Scarabaeidae) in unfavourable cattle dung. Bulletin of Entomological Research 80:473-478.

Rodrigues, M. M. 2008. Besouros coprófagos (Coleoptera: Scarabaeoidea) em três diferentes usos do solo no sul de Mato Grosso do Sul, Brasil. Dissertação de Mestrado. Dourados, Universidade Federal da Grande Dourados.

Rodrigues, S. R. \& Flechtmann, C. A. H. 1997. Aspectos biológicos de Canthon lituratus (Germar, 1813) e Canthidium (Canthidium) megatophoides Boucomont, 1928 (Coleoptera, Scarabaeidae). Acta Zoológica Mexicana 70:1-12.

Rodrigues, S. R.; Marchini, L. C. \& Carbonari, J. J. 2001a. Ácaros das famílias Scutacaridae e Pygmephoridae (Acari: Heterostigmata) associados a besouros coprófagos (Coleoptera: Scarabaeidae) no Brasil. Neotropical Entomology 30(3):387-390. 
Rodrigues, S. R.; Marchini, L. C. \& Mendes, M. C. 2001b. Ácaros da família Macrochelidae (Gamasida) associados com besouros coprófagos (Scarabaeidae). Revista Brasileira de Entomologia 45(3):207-214.

Rodrigues, S. R.; Barros, A. T. M.; Puker, A. \& TAira, T. L. 2010 Diversidade de besouros coprófagos (Coleoptera, Scarabaeidae) coletados em armadilha de interceptação de vôo no Pantanal SulMatogrossense, Brasil. Biota Neotropica 10(2):123-127.

VAZ-DE-MELlo, F. Z. 2000. Estado atual de conhecimento dos Scarabaeidae s. str. (Coleoptera: Scarabaeoidea) do Brasil.. In: Martín-Piera, F.; Morrone, J. J. \& Melic, A. eds. Hacia un Proyecto Cyted para el Inventario y Estimación de la Diversidad Entomológica en Iberoamérica. Zaragoza, PrIBES-SEA, p. 183-195.

VAZ-DE-Mello, F. Z. 2007a. Revisión taxonómica y análisis filogenético de la tribu Ateuchini (Coleoptera: Scarabaeidae: Scarabaeinae).
Tesis de Doctorado en Ciencias Sistematica. Xalapa, México Instituto de Ecología A. C. 237p.

VAZ-DE-Mello, F. Z. 2007b. Revision and Phylogeny of the dung beetle genus Zonocopris Arrow, 1932 (Coleoptera: Scarabaeidae: Scarabaeinae), a phoretic of land snails. Annales de la Société Entomologique de France, Nouvelle série 43(2):231-239.

VAZ-DE-Mello, F. Z. 2008. Synopsis of the new subtribe Scatimina (Coleoptera: Scarabaeidae: Scarabaeinae: Ateuchini), with descriptions of twelve new genera and review of Generidium, new genus. Zootaxa 1955:1-75.

VAZ-DE-Mello, F. Z. \& Grossi, P. C. 2010. First report of Bolbites Harold (Scarabaeidae: Scarabaeinae: Phanaeini) in Brazil. The Coleopterists Bulletin 64(3):220.

Waterhouse, D. F. 1974. The biological control of dung. Scientific American 230:101-108. 